\title{
Erratum to: A comprehensive study of the resource discovery techniques in Peer-to-Peer networks
}

\author{
Nima Jafari Navimipour • Farnaz Sharifi Milani
}

Published online: 21 February 2015

(C) Springer Science+Business Media New York 2015

Erratum to: Peer-to-Peer Netw. Appl.

DOI 10.1007/s12083-014-0271-5

In the original version of this Review Paper, the affiliations of the authors are incorrect. Corrected affiliations of the authors are given here.

The online version of the original article can be found at http://dx.doi.org/ 10.1007/s12083-014-0271-5.

N. Jafari Navimipour $(\bowtie)$

Department of Computer Engineering, Tabriz Branch, Islamic Azad

University, Tabriz, Iran

e-mail: Jafari@iaut.ac.ir

F. Sharifi Milani

Young Researchers and Elite Club, Tabriz Branch, Islamic Azad

University, Tabriz, Iran 